\title{
The COM-B System of Behaviour Change: Properties, Problems and Prospects
}

\author{
David F Marks
}

Funding: The author(s) received no specific funding for this work.

Potential competing interests: The author(s) declared that no potential competing interests exist.

\begin{abstract}
The COM-B system of behaviour change is analysed. The system involves three processes termed "Capability", "Opportunity" and "Motivation". The crucial motivational element of "Wanting" is missing from the system. Wanting eliminates the intention-behaviour gap in explaining why people do or do not do the things necessary for their survival. Prospects for self-protective behaviour change in health emergencies such as the SARS-COV-2 pandemic will be greatly improved when the core motivational process of wanting is restored within motivational theory.
\end{abstract}

\section{Definitions}

Capability

Defined by Susan Michie et al.

\section{Motivation}

Defined by Susan Michie et al.

\section{Opportunity}

Defined by Susan Michie et al.

\section{Want}

Defined by David F Marks

Need

Defined by David F Marks

Human choices and methods for influencing them are core topics in theoretical psychology. In attempting to mitigate the effects of the SARS-COV-2 pandemic, it is more than an academic issue to determine whether current models of behaviour change are fit for purpose. A major class of models termed 'social-cognitive models' (SCM) rely on social and cognitive representations that are hypothesised to take place inside people's heads. In spite of their widespread use, neither the prenor post-SARS-COV-2 evidence on behaviour change justifies any confidence in the SCMs. The evidence suggests that current psychological theories and models are an inadequate foundation for effective interventions. A critique of the main SCMs has been published elsewhere (Marks, Murray \& Estacio, 2018). The majority of SCMs show an individualistic bias, internalize choice as a process similar to the operating system of a computer, assumed that the processes are both universal and rational by following a fixed set of formulae that the models attempt to describe. In using small samples of 
college students or patients, the power, ecological validity and generalizability of the majority of studies is questionable. Multiple procedural issues indicate that the standard procedure for developing questionnaires used in theory testing systematically produce problematic questions. Most studies use self-reported measures of intention and behaviour rather than objective measures. The models generally neglect culture, ethnicity, religion and gender. SCMs do not adequately address the motivational determinants of risky behaviours. Contrary to the SCMs' assumptions, in certain cases, the very riskiness of certain behaviours is responsible for their adoption.

On the basis of current evidence, SCMs and other theories claiming universal application receive weak empirical support in studies of behaviour change. In spite of the lack of supportive evidence, one recent model called the 'CapabilityOpportunity-Motivation Behaviour' moeld or 'COM-B' has been advocated in a variety of behavioural domains including personal protective behaviours to prevent infection during the SARS-CoV-2 outbreak. Michie, van Stralen and West (2011) synthesised interventions into a single framework with two levels, one representing intervention functions and the other representing higher-order policy categories. The resulting 'Behaviour Change Wheel' is claimed by its inventors to provide "a systematic way of characterising interventions that enables their outcomes to be linked to mechanisms of action, and can help to diagnose why an intervention may have failed to achieve its desired goal. The nine intervention functions and seven policy categories are linked to a model of behaviour at the hub of the wheel" (Michie, Atkins \& West, 2014), which is the COM-B. I review here the nature of the COM-B system, its properties, problems and prospects. The COM-B System

Michie, Van Stralen and West (2011) proposed "a 'behaviour system' involving three essential conditions: capability, opportunity, and motivation" that forms the hub of the 'Behaviour Change Wheel' (BCW)." The authors mention two sources for the idea of the COM-B:

"a US consensus meeting of behavioural theorists in 1991 [see Fishbein et al., 2001] and a principle of US criminal law dating back many centuries...Under US criminal law, in order to prove that someone is guilty of a crime one has to show three things: means or capability, opportunity, and motive."

They continue:

"This suggested a potentially elegant way of representing the necessary conditions for a volitional behaviour to occur...We have built on this to add nonvolitional mechanisms involved in motivation (e.g., habits) and to conceptualise causal associations between the components in an interacting system."

A conceptual framework used by the courts to prove the innocence or guilt of accused felons may not necessarily be the most appropriate model to apply to the everyday behavioural choices of non-criminals. In spite of the widespread attention paid to the COM-B, in the analysis to be presented, the hub of the COM-B is incomplete and manifestly does not work when applied to the most basic of choices such as smoking, drinking and the wearing of face masks in a pandemic. Here I explain why. 


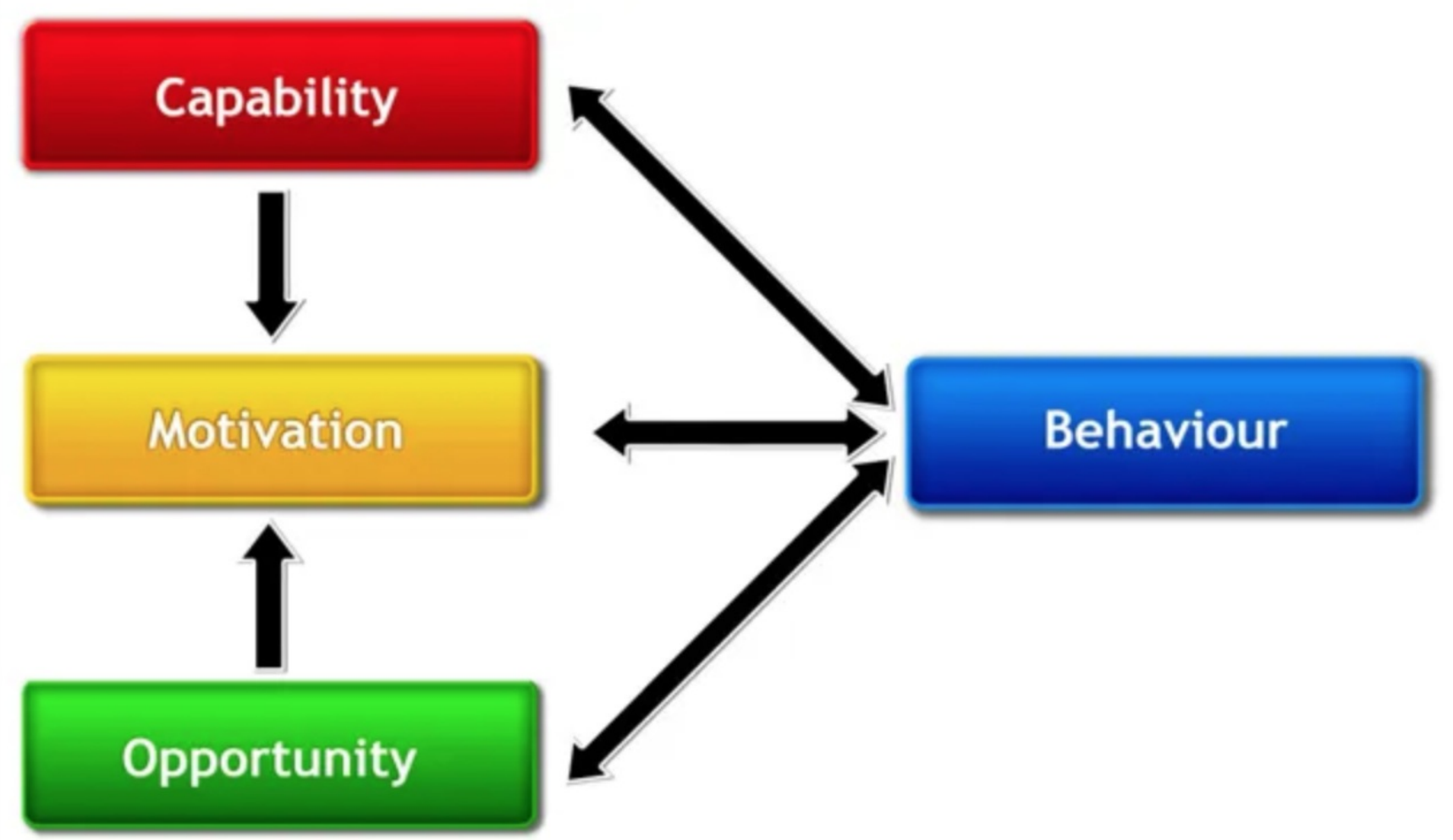

\section{The COM-B system - a framework for understanding behaviour.}

In the COM-B system, Capability, Opportunity, and Motivation are said to 'interact' to generate Behaviour. C, O and M are claimed to be sufficient and necessary conditions for B. Before discussing the model, we need some definitions:

\section{Definitions}

Michie et al. (2011) give the following definitions:

'Capability' is defined as the individual's psychological and physical capacity to engage in the activity concerned. This includes having the necessary knowledge and skills. In plain language, 'capability' means 'fit to'.

'Motivation' is defined as all those brain processes that energize and direct behaviour, not just goals and conscious decision-making, e.g. habitual processes, emotional responding, as well as analytical decision-making. Thus motivation equates with 'need to'.

'Opportunity' is defined as all of the factors that 'lie outside the individual that make the behaviour possible or prompt it'.

Thus opportunity means 'can do'.

To these definitions, it is necessary to add definitions for 'want' and 'need':

'Want' - A mental state that motivates an individual or group towards a goal that is desirable but inessential for survival.

"Need" - A physical or mental state that motivates an individual or group towards a goal that is desirable and essential for survival.

Note that 'want' and 'need' are overlapping but different constructs so that it is possible to want without need (e.g. to go to the cinema) or to need without want (e.g. to go to the dentist). One can simultaneously both need and want something (e.g. to go to the supermarket) or both not need and not want (e.g. to go into quarantine when travelling into the UK during the 
pandemic). All four scenarios exist when capability and opportunity are both present. Clearly, this is a major logical problem for the COM-B system and the BCW. If the hub is broken, then the wheel cannot work either.

The authors of the COM-B model claim that if one is fit to, needs to and can do a particular behaviour, then one will do it. They claim causal connections between the $\mathrm{C}, \mathrm{O}$ and $\mathrm{M}$ components that cannot possibly exist. As indicated above, the simplistic three-pronged approach of the COM-B cannot possibly work because the process of wanting is missing. Using the original context for the COM-B model - the proving of guilt or innocence in the courtroom - the following example illustrates the fundamental problems with the COM-B.

Consider the example drawn from the domain of criminology: Robbing a Bank. I refer to that fictitious character Joe Blow (pronouns: him/her/their). According to the COM-B: Joe Blow (JB) would (X rob a bank, Y kiss the queen, $Z$ fly to the moon, whatever) if it can be shown that JB is fit to, needs to, and can do $X, Y$ or $Z$. The key missing element of wanting is the determining factor when the other three factors are all present. JB must want to carry out $X, Y$ or $Z$ if he is to actually to do it. If JB doesn't want to, he/she/they simply won't do it, no matter what.

Imagine the following scenario:

1) Capability: JB is fit to rob a bank because JB is physically strong and has a jemmy and a set of tools for breaking open doors and safes.

2) Opportunity: JB has discovered that there is a back alley and a back door with an alarm that a friend who works in the bank will leave switched off on any night of their choosing.

3) Motivation: JB is hugely in debt (to a bank, as it happens) so he/she/they need(s) money very badly, and so there is a strong motive to rob a bank.

However, in spite of ticking all three boxes of the COM-B, JB chooses not to rob the bank. Why so? It could be for a million-and-one different reasons. JB does not rob the bank because:

- robbing the bank would be wrong,

- robbing the bank would be risky - i.e. if JB is convicted of the crime, JB would go to prison,

- if found out, it would look bad in front of the neighbours,

- robbing the bank would upset the bank manager whom JB knows well and meets for drinks in the local pub,

- robbing the bank would upset JB's partner,

- robbing the bank would be an unreasonable and unfair,

- etc

In spite of JB ticking all three of the COM-B boxes, the COM-B fails to predict JB's behaviour. There is a hidden barrier. In multiple situations people do not choose to do something from which they could not only benefit, and perhaps even avoid a catastrophic outcome including their own death, simply because they do not want to.

Returning to the example, imagine another similar individual, JB's twin, who ticks all three of the COM-B boxes and proceeds to commit the bank robbery. JB's twin, Les Blow (LB), lives on the other side of town. JB tells LB about the bank, the back alley and the dodgy security guard. Like JB, LB meets all three COM-B criteria - fit to, needs to, and can do the bank robbery. However, LB has none of JB's moral and social scruples and LB proceeds to rob the bank. The twins act differently under essentially similar circumstances, revealing the crucial importance of wanting.

The COMA-B 
The above considerations indicate that the COM-B requires reformulation. The diagram of the COM-B model shows five arrows representing so-called 'interactions', three of which point in both directions. However, four of the 'interactions' do not exist and none works in both directions. Only one 'interaction' in the COM-B diagram is anywhere near causal - motivation does indeed cause behaviour. However, motivation includes both needs and wants. As we have shown, in order to do something, a person has to want to do it more than they want not to do it. The COM-B is reformulated as the 'COMA-B' in the diagram below. [A stands for 'Aspires to', which produces a better acronym than using W for 'Wants' : COMW-B].

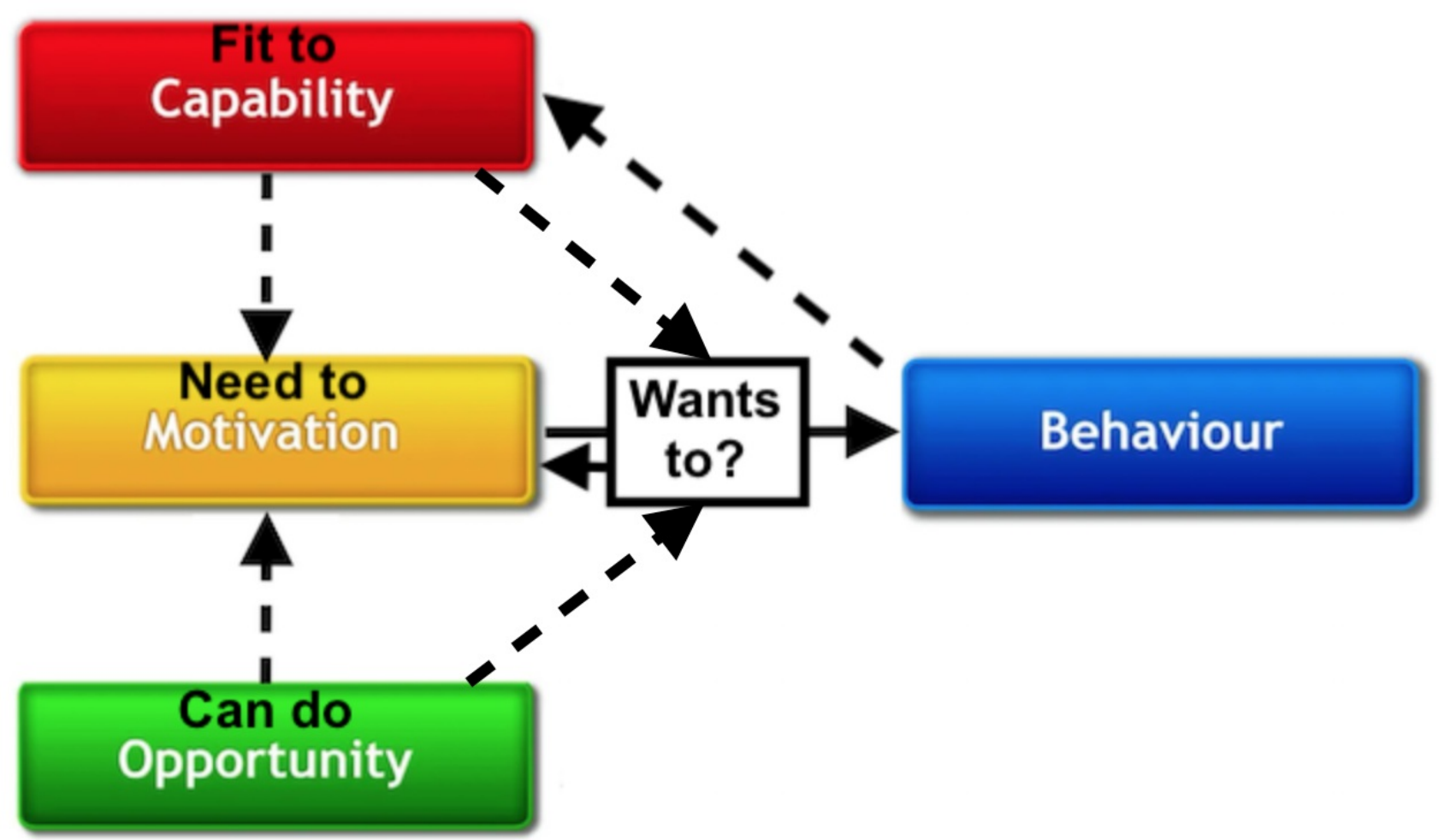

The COMA-B system includes the crucial motivational process of Wanting. Wanting has a direct causal function (solid arrows) on behaviour and can bypass Need. Capability and Opportunity both have an enabling function (broken arrows) not a causal function.

Adding a box for 'wants to' and using two types of arrows to separate enablers from causes converts the 'COM-B' into the 'COMA-B'. In the COMA-B system, 'fit-to' capability and 'can-do' opportunity are enablers; 'want-to' is causal. Not wanting to do something can block or reduce the influence of need. Wanting-to-do something converts opportunities and capabilities into actions, even when need is missing.

In sum, three necessary conditions for any action $\mathrm{X}$ are:

1. having the capability to do $X$ enables $X$

2. having the opportunity to do $X$ enables $X$

3. wanting to do $X$ causes $X$

A fourth condition, needing to do $\mathrm{X}$, is neither necessary nor sufficient to produce $\mathrm{X}$ unless wanting to do $\mathrm{X}$ is also present. Reformulation of the COM-B

In a recent publication, West and Michie. (2020) have reformulated the COM-B system bringing it more into line with logical and empirical requirements. Several features of the new COM-B-V2 are noted: "First, capability and opportunity are shown as influencing the relationship between motivation and behaviour, rather than the behaviour itself. This represents the idea 
that, at an individual and moment-to-moment basis, they act like 'logic gates' in that both of the 'gates' (capability and opportunity) need to be open for motivation generate the behaviour. Aggregated over time and people, we can think of capability and opportunity more quantitatively: the greater the capability and opportunity the more likely a behaviour is to occur because the more often the 'gates' will be open when the motivation is present" (West \& Michie, 2020). Capability and opportunity have become 'gates', or what I have termed above, 'enablers'. However, the COM-B-V2 retains the fatal flaw of omitting wants as the crucial causal determinant of behaviour. Wanting converts intention into behaviour and removes the intention-behaviour gap.

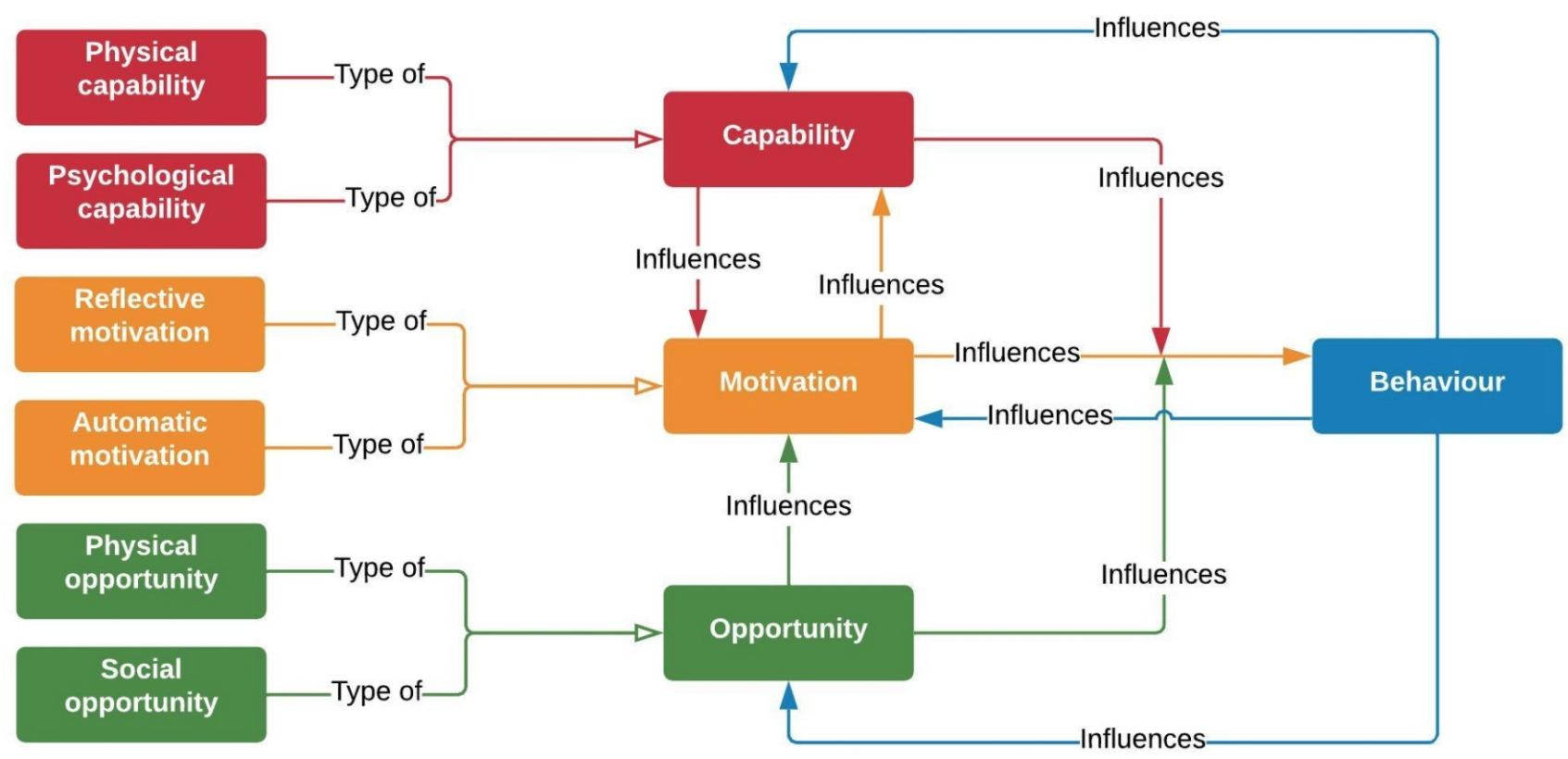

\section{CONCLUSIONS}

1. The COM-B and COM-B-V2 are dysfunctional as hubs for behaviour change interventions because they omit the crucial causal role played by wanting.

2. For the above reason, the COM-B and COM-B-V2 models of behaviour change are unfit for purpose. The failure to include wanting explains the intention-behaviour gap and the lack of empirical support for models of behaviour change.

3. Prospects for behaviour change models in health emergencies such as the SARS-COV-2 pandemic will be greatly improved when the core motivational process of wanting is restored within motivational theory.

\section{REFERENCES}

Fishbein, M., Triandis, H. C., Kanfer, F. H., Becker, M., Middlestadt, S. E., \& Eichler, A. (2001). Factors influencing behavior and behavior change. Handbook of health psychology, 3 ,

Marks, D.F., Murray, M. \& Estacio, E.V.G. (2018). Health psychology. Theory, research \& Practice (6 ${ }^{\text {th }}$ ed.). London: Sage Publications.

Michie, S., Atkins, L., \& West, R. (2014). The behaviour change wheel. A guide to designing interventions. 1st ed. Great 
Britain: Silverback Publishing, 1003-1010.

Michie, S., Van Stralen, M. M., \& West, R. (2011). The behaviour change wheel: a new method for characterising and designing behaviour change interventions. Implementation science, 6(1), 42.

West, R. \& Michie, S. (2020). A brief introduction to the COM-B Model of behaviour and the PRIME Theory of motivation. Qeios. doi:10.32388/WW04E6.2. 